\title{
Measurement and validation of PbS nanocrystal energy levels
}

\author{
D. M. N. M. Dissanayake, ${ }^{\text {a) }}$ T. Lutz, R. J. Curry, and S. R. P. Silva \\ Advanced Technology Institute, Faculty of Engineering and Physical Sciences, University of Surrey, \\ Surrey GU27XH, United Kingdom
}

(Received 26 May 2008; accepted 7 July 2008; published online 28 July 2008)

\begin{abstract}
Cyclic voltammetry was used to measure PbS nanocrystal (PbS-NC) energy levels. Accuracy of these measurements was justified by electron transfer experiments, with well known fullerene derivatives, which included photoluminescence quenching experiments and current density-voltage measurements of hybrid photovoltaic devices. It is believed that these energy level measurements, carried out on PbS-NCs under ambient conditions, would provide valuable information for the design and fabrication of optimized hybrid nanoelectronic devices. (C) 2008 American Institute of Physics. [DOI: 10.1063/1.2964203]
\end{abstract}

Narrow bandgap colloidal semiconductor nanocrystals (NCs) are actively researched for their size depended optical properties and solution processibility. ${ }^{1,2}$ NCs can be incorporated with organic semiconductors to fabricate low cost, flexible, and large area optoelectronics and photonic hybrid devices. ${ }^{3,4}$ A fundamental drawback in the design of hybrids is the accurate understanding of energy level positions, which govern charge transfer at NC-organic interfaces. Mostly, NC band diagrams reported in literature for hybrid devices are obtained by calculations, based on bulk ionization potential (IP) and electron affinity (EA). ${ }^{1}$ Direct measurement of the IP, using photoemission spectroscopic techniques, has been reported for PbS-NCs. ${ }^{3}$ However, a major problem encountered in such studies was the detrimental effect caused by the passivating organic ligands, capping the $\mathrm{NCs}$, on photoemission measurements and subsequent analysis. Insulating organic ligands caused charge buildup on the substrates as well as screened NCs from exposure to ultraviolet photons, severely affecting the accuracy of the measurements. Consequently, these NCs were annealed in ultrahigh vacuum to overcome such problems and the calculated energy levels showed reasonable agreement to the device performance. However, these annealing techniques were reported to alter the surface (bulk) condition as well as the stoichiometry of the NCs from its condition present at device fabrication. ${ }^{3}$ Therefore, less invasive techniques such as electrochemistry have to be employed for measurement of these NC energy levels. Kucur et al. reported IP and EA values for different size CdSe NCs using cyclicvoltammetry. ${ }^{5}$ Furthermore, differential pulse voltammetry, which demonstrate greater sensitivity than cyclicvoltammetry, has been used previously for determining the subtle changes of the NC energy levels, with different ligands, proving the versatility of electrochemical techniques. ${ }^{6}$ In this paper, we utilize a technique of electrochemistry to investigate the energy levels of PbS-NCs and probe the accuracy of the obtained values by charge transfer experiments. Current density-voltage $(J V)$ characteristics of hybrid photovoltaic (PV) devices fabricated using $\mathrm{C}_{60}$ and soluble $\mathrm{C}_{60}$ derivative of $[6,6]$-phenyl- $\mathrm{C}_{61}$-butyric acid methyl ester $(\mathrm{PCBM})$, and photoluminescence (PL) quenching experiments were used for determining the electron transfer (ET) from PbS-NCs

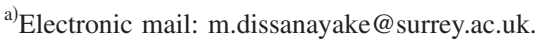

justifying the energy band diagrams obtained. This work provides crucial information about PbS-NC energy levels for the design and fabrication of optimized hybrid optoelectronic devices.

$\mathrm{PbS}-\mathrm{NCs}$ with rocksalt crystalline structure were synthesized according to a method reported by Hines and Scholes. ${ }^{7}$ Approximate size of these PbS-NCs was calculated as 3.8 $\mathrm{nm}$ by applying the absorption onset value to theoretical models documented elsewhere. ${ }^{8}$ All chemicals were analytical grade reagents and were purchased from Sigma Aldrich and used as received. As-synthesized capping oleic acid ligands were exchanged for shorter butylamine ligands ${ }^{2}$ and $\mathrm{PbS}-\mathrm{NCs}$ were dissolved in anhydrous toluene $(70 \mathrm{mg} / \mathrm{ml})$. Electrochemical measurements were carried out using an Autolab PGSTAT12 potentiostat/galvanostat. The PbS-NC solution was drop casted and dried on top of $1 \mathrm{~cm}^{2}$ area $\mathrm{Pt}$ electrode for measurements. $0.1 M$ tetrabutylammoniumhexaflurophosphate $\left(\mathrm{TBAPF}_{6}\right)$ dissolved in acetonitrile (HPLC grade) was used as the electrolyte solution. A homemade $\mathrm{Ag} / \mathrm{AgCl}$ electrode, calibrated against ferrocene [0.5 $\mathrm{mM}$ in acetonitrile:TBAPF $6(0.1 M)]$ solution, was used as the reference electrode. Cyclicvoltammetry sweeps were carried out at $50 \mathrm{mV} / \mathrm{s}$, starting from negative to positive bias, with the electrochemical cell purged with nitrogen for 10 min prior to each measurement. Hybrid PVs were fabricated by spin coating $\mathrm{PbS}-\mathrm{NC}$ solutions $(70 \mathrm{mg} / \mathrm{ml})$ on poly $(3,4-$ ethylenedioxythiophene)-poly(styrenesulfonate) coated indium tin oxide substrates to obtain uniform film thickness of $100 \mathrm{~nm} .30 \mathrm{~nm} \mathrm{C} 60$ (99.99\%, American Dye Source) was sublimated on PbS-NC film and afterwards a $10 \mathrm{~nm}$ bathocuproine (BCP) and $50 \mathrm{~nm} \mathrm{Al}$ top contact deposited to fabricate $10 \mathrm{~mm}^{2}$ PVs. Similarly, a film of PCBM $(20 \mathrm{mg} / \mathrm{ml}$ in $\left.\mathrm{CHCl}_{3}\right)(99.9 \%$, Solenne) was spin coated at $700 \mathrm{rpm}(60 \mathrm{~s})$ on predeposited $\mathrm{PbS}-\mathrm{NCs}$ and subsequently capped by a $\mathrm{BCP}$ and $\mathrm{Al}$ top contact to fabricated PbS-NC:PCBM device. $J V$ characteristics of the devices were obtained using an Oriel solar simulator at AM1.5G simulated solar irradiation. $\mathrm{PL}$ experiments were carried out on PbS-NC: $\mathrm{C}_{60}$ and $\mathrm{PbS}$ NC:PCBM bilayer thin film samples to study effects of PL quenching resulting from ET from $\mathrm{PbS}-\mathrm{NCs}$ to the fullerene derivatives. An Ar ion laser operating at $351 \mathrm{~nm}(150 \mathrm{~mW})$ UV was used for the PL experiments.

Cyclicvoltammogram taken of PbS-NCs deposited on a Pt electrode is shown in Fig. 1 with voltage taken relative to 


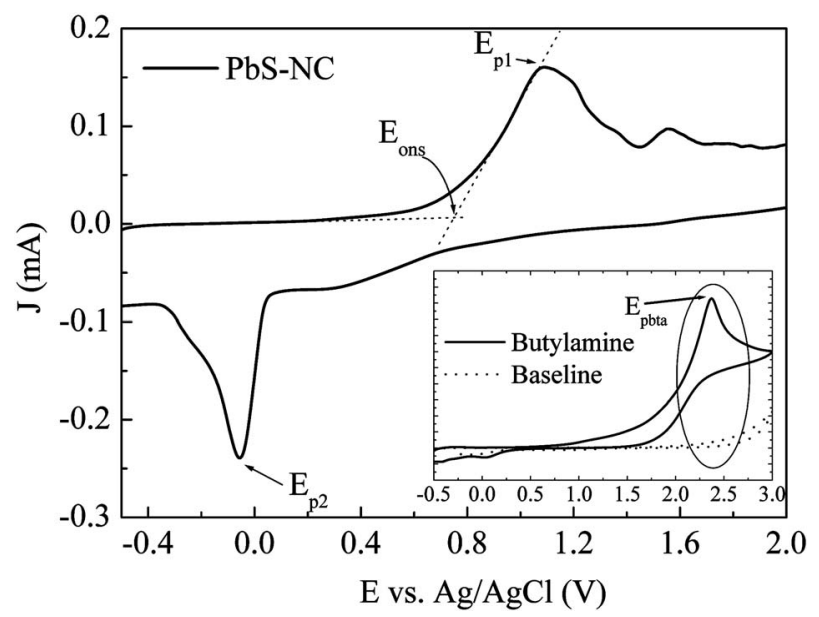

FIG. 1. Cyclicvoltammetry graph of PbS-NC film deposited on the working

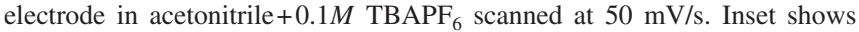
the voltammograms of $5 \mathrm{mM}$ of butylamine (solid line) and a baseline taken (dotted line) on acetonitrile $+0.1 M \mathrm{TBAPF}_{6}$ pristine solution.

the reference electrode. Distinct peaks $E_{p 1}$ and $E_{p 2}$ of the voltammogram are seen at 1.08 and $-0.06 \mathrm{~V}$ in the forward and reverse cycles, respectively. Both $E_{p 1}$ and $E_{p 2}$ can clearly be distinguished from baseline, shown by the dotted line in inset Fig. 1, thus can be attributed to the Faradic processes occurring in the PbS-NC sample. To clearly separate the redox reactions taking place in the butylamine ligands from $\mathrm{PbS}-\mathrm{NC}$ reactions, a reference voltammogram was carried out on the ligands (solid line in the Fig. 1 inset). A redox peak $\left(E_{\mathrm{pbta}}\right)$ of the butylamine voltammogram seen at $2.37 \mathrm{~V}$ can be clearly separated from the $E_{p 1}$ and $E_{p 2}$ seen for the $\mathrm{PbS}-\mathrm{NC}$ sample. Hence, it can be concluded that the $E_{p 1}$ and $E_{p 2}$ corresponds to the oxidation and reduction reactions respectively of the PbS-NCs only. Using above measurements, IP of the PbS-NCs was calculated using the onset potential $\left(E_{\text {ons }}\right)$ and reference electrode potential against the vacuum level $\left(I_{\mathrm{RE}}\right)$, according to Eq. $(1), 5,9,10$

$$
\mathrm{IP}=-\left(E_{\text {ons }}+I_{\mathrm{RE}}\right)[\mathrm{eV}] \text {. }
$$

$I_{\mathrm{RE}}$ was obtained as $4.48 \mathrm{eV}$ by calibration against the oxidation potential of ferrocene (Ref. 11) (0.5 mM in acetonitrile $+0.1 M \mathrm{TBAPF}_{6}$ ) Therefore the IP is calculated as $5.2 \mathrm{eV}$ for PbS-NCs using $E_{\text {ons }}$ value $0.75 \mathrm{~V}$ measured from Fig. 1. As previously reported, onset reductions potentials were not seen to provide accurate values for the EA of NCs and therefore not used in this study. ${ }^{5}$ Alternatively, EA is obtained by subtracting the optical bandgap $(1.2 \mathrm{eV})$, obtained from PbS-NC optical absorption onset (Fig. 2), from the calculated IP value. Therefore, the EA value for the PbSNCs used for this study is taken as $(4.0 \mathrm{eV})$. It should be noted that the size distribution of the PbS-NCs sample ( $\sim 10 \%)$, derived from the full width at half maximum (0.186 $\mathrm{eV})$ of the PL spectra given in Fig. 2, introduces an error to the EA value $(< \pm 0.1 \mathrm{eV})$ calculated above. Quantification of the error introduced by PbS-NCs size distribution on the derivation of the IP value is non trivial and therefore differed for a more detailed study.

Using the IP and EA obtained above, energy diagrams can be proposed as schematically, as shown in inset Fig. 2, between PbS-NCs and $\mathrm{C}_{60}$ (PCBM) donor-acceptor hybrids. $\mathrm{C}_{60}$ and PCBM are very well characterized electron acceptors, extensively used in organic PVs. Since the EA, $4.5 \mathrm{eV}$

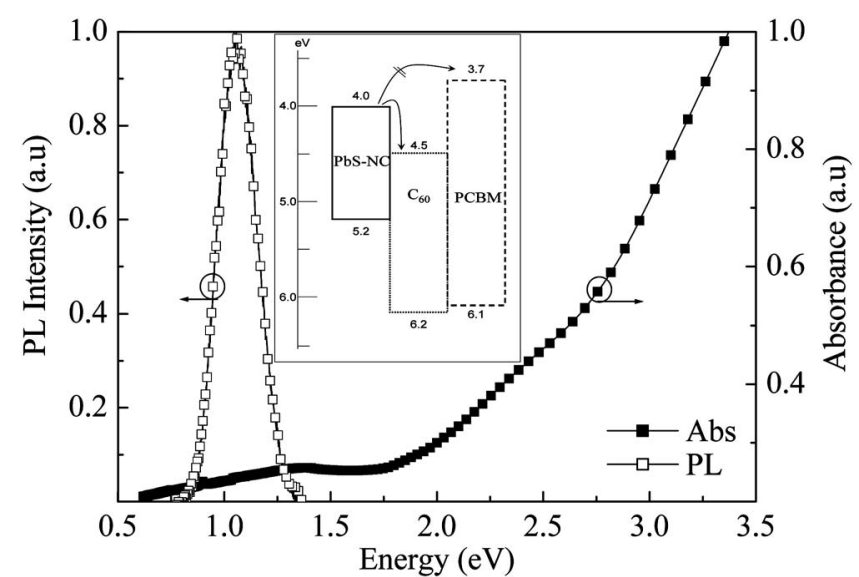

FIG. 2. Absorbance (black square) and PL (white square) of PbS-NCs spin coated on quartz. Inset shows the energy diagrams between bilayer systems of $\mathrm{PbS}-\mathrm{NCs}$ and the fullerene derivatives.

(3.7 eV), and IP, $6.2 \mathrm{eV}(6.1 \mathrm{eV})$, of $\mathrm{C}_{60}$ (Ref. 12) (PCBM) (Ref. 13) are well known, they can be used effectively as references in charge transfer experiments. Observing the above band diagram, it is possible to infer the rate of ET between PbS-NCs and $\mathrm{C}_{60}$ (PCBM) layers. ET from PbS$\mathrm{NCs}$ to $\mathrm{C}_{60}$ is seen to be more energetically favorable as compared to ET from PbS-NCs to PCBM. Under flat band condition there exist a $0.3 \mathrm{eV}$ energy barrier between the NCs and PCBM obstructing the ET.

To probe qualitatively the ET between PbS-NCs and $\mathrm{C}_{60}$ (PCBM) and justify the band diagrams obtained above, PL experiments were carried out. Ultrafast charge transfer at a donor-acceptor interface is known to significantly quench radiative recombination of the donor and, therefore, is a widely used technique in molecular electronics. ${ }^{14}$ Bilayer PbS-NC: $\mathrm{C}_{60}$ (PCBM) films were excited and the PbS-NC PL was observed. As seen from Fig. 3, PbS-NC PL is significantly quenched in the presence of $\mathrm{C}_{60}$ proving efficient ET consistent with the above hypothesis. Approximately two orders greater PL intensity is observed between PbS-NC:PCBM bilayer as compared to $\mathrm{PbS}-\mathrm{NC}: \mathrm{C}_{60}$ indicating greater radiative recombination, attributed to reduced ET from PbS-NCs to PCBM. As shown in Fig. 3, single layer of PCBM did not demonstrate PL at the wavelength range similar to

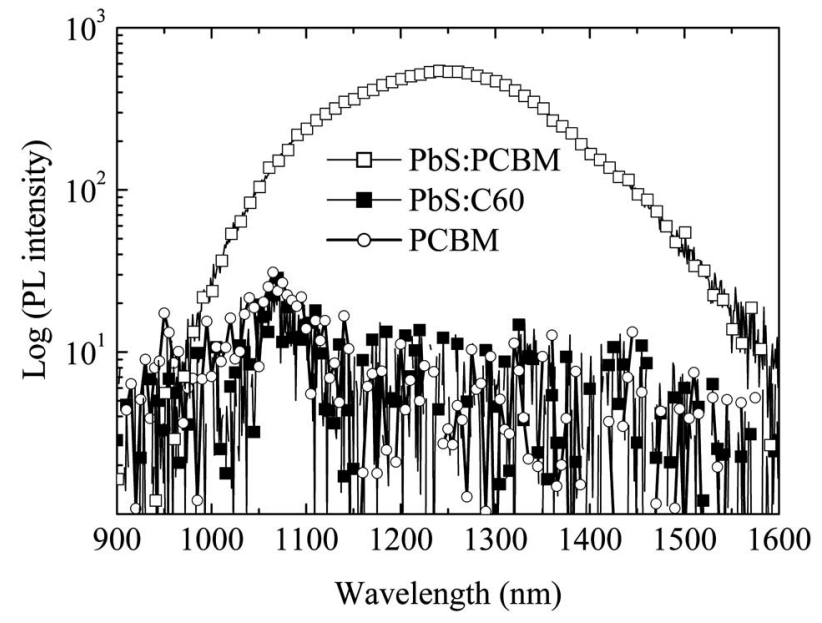

FIG. 3. PL intensities of PbS-NC:PCBM (white squares), PbS-NC: $\mathrm{C}_{60}$ (black squares), and PCBM only (white circles) excited using $351 \mathrm{~nm} \mathrm{Ar}$ ion laser. 


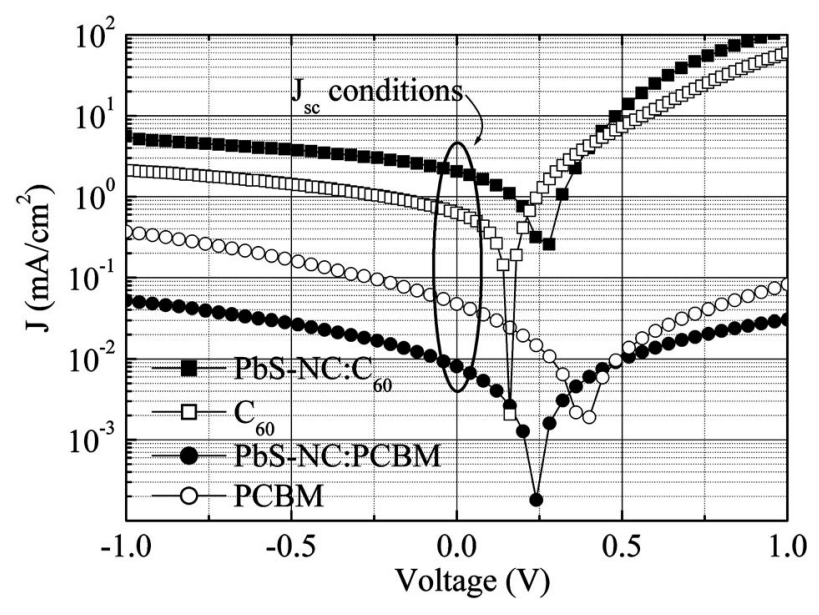

FIG. 4. $J V$ characteristics of PbS-NC: $\mathrm{C}_{60}$ (black squares), $\mathrm{C}_{60}$ single layer (white squares), PbS-NC:PCBM (black circles) and PCBM single layer (white circles) devices.

PbS-NC:PCBM sample, further confirming above observations.

To confirm accuracy of the energy level calculations and results obtained from PL quenching, hybrid PVs where fabricated using $\mathrm{PbS}-\mathrm{NC}$ and the fullerene (derivatives) used above (Fig. 4). The PbS-NC: $\mathrm{C}_{60}$ device shows larger short circuit current density $\left(J_{\mathrm{sc}}\right)$ as compared to the reference $\mathrm{C}_{60}$ single layer device. This increase of $J_{\mathrm{sc}}$ is attributed to the increased photon harvesting of PbS-NCs together with the increased exciton dissociation at the heterojunction. Therefore, it can be concluded that reasonably efficient ET exist between the PbS-NCs and $\mathrm{C}_{60}$. Conversely, the $J_{\mathrm{sc}}$ of $\mathrm{PbS}-\mathrm{NC}: \mathrm{PCBM}$ is nearly an order of magnitude lower than the $J_{\mathrm{sc}}$ of PCBM single layer device clearly attributed to poor ET at PbS-NC:PCBM heterojunction as compared to the $\mathrm{PbS}-\mathrm{NC}: \mathrm{C}_{60}$. The PbS-NC layer can also absorb a significant amount of light going through to the PCBM, subsequently reducing the number of photons harvested in the PCBM layer. Overall, the decreased ET reduces net current generated though the PbS-NC:PCBM device compared to PCBM single layer device. Furthermore, PbS-NC: $\mathrm{C}_{60}$ shows greater than two orders of magnitude larger $J_{\mathrm{sc}}$ as compared to PbS-NC:PCBM agreeing with the PL quenching data shown in Fig. 3. Therefore, both PL quenching experiments and the $J V$ characteristics of $\mathrm{PbS}-\mathrm{NCs}$ and $\mathrm{C}_{60}$ (PCBM) favorably justifies the band diagram obtained from cyclicvoltammetry measurements discussed above. It can also be postulated that majority of charge transfer occurs from band-edge energy levels as opposed to the higher energy levels. This has important implication for hybrid device fabrication using $\mathrm{NCs}$ in general. For example, promising NC based PVs proposed in literature assume hot electron injection from higher energy levels from the NCs to acceptors. Clearly such hot electron injection was not seen to manifest in the device architectures studied here.

In conclusion, cyclic voltammetry was used measure the EA and IP of PbS-NCs in ambient conditions. The accuracy of these measurements was justified by ET experiments with well characterized acceptor materials $\left[\mathrm{C}_{60}(\mathrm{PCBM})\right]$, which included PL quenching experiments and $J V$ measurements of hybrid PVs. It is believed that these energy levels measurements obtained and verified, using the techniques described above, would provide valuable information for the design and fabrication of optimized hybrid nanoelectron devices in the future.

The authors would like to acknowledge the valuable information proved by Professor Thomas Nann for the cyclicvoltammery experiments. Furthermore, the funding provided by EPSRC portfolio grant and First grant is also generously acknowledged.

${ }^{1}$ S. A. Mcdonald, G. K. Konstantatos, S. Zhang, P. W. Cyr, E. J. D. Klem, L. Levina, and E. H. Sargent, Nat. Mater. 4, 138 (2005).

${ }^{2}$ G. Konstantatos, I. Howard, A. Fischer, S. Hoogland, J. Cliford, E. Klem, L. Levina, and E. H. Sargent, Nature (London) 442, 180 (2006).

${ }^{3}$ D. M. N. M. Dissanayake, R. A. Hatton, T. Lutz, C. E. Giusca, R. J. Curry, and S. R. P. Silva, Appl. Phys. Lett. 91, 133506 (2007).

${ }^{4}$ K. N. Bourdakos, D. M. N. M. Dissanayake, T. Lutz, S. R. P. Silva, and R. J. Curry, Appl. Phys. Lett. 92, 153311 (2008).

${ }^{5}$ E. Kucur, J. Riegler, G. A. Urban, and T. Nann, J. Chem. Phys. 119, 2333 (2003).

${ }^{6}$ M. Soreni-Harari, N. Yaccobi-Gross, D. Steiner, A. Aharoni, U. Banin, O. Millo, and N. Tessler, Nano Lett. 8, 678 (2008).

${ }^{7}$ M. A. Hines and G. D. Scholes, Adv. Mater. (Weinheim, Ger.) 15, 1844 (2003).

${ }^{8}$ L. Cademartiri, E. Montanari, G. Calestani, A. Migliori, A. Guagliardi, and G. A. Ozin, J. Am. Chem. Soc. 128, 10337 (2006).

${ }^{9}$ J. L. Bredas, R. Silbey, D. S. Boudreaux, and R. R. Chance, J. Am. Chem. Soc. 105, 6555 (1983).

${ }^{10}$ S. Janietz, D. D. C. Bradley, M. Grell, C. Giebeler, M. Inbasekaran, and E. P. Woo, Appl. Phys. Lett. 73, 2453 (1998).

${ }^{11}$ A. M. Bond, K. B. Oldham, and G. A. Snook, Anal. Chem. 72, 3492 (2000).

${ }^{12}$ A. Yakimov and S. R. Forrest, Appl. Phys. Lett. 80, 1667 (2002).

${ }^{13}$ T. W. Lee, Y. Byun, B. W. Koo, I.-N. Kang, Y. Y. Lyu, C. H. Lee, L. Pu, and S. Y. Lee, Adv. Mater. (Weinheim, Ger.) 17, 2180 (2005).

${ }^{14}$ D. S. Ginger and N. C. Greenham, Phys. Rev. B 59, 10622 (1999). 